\title{
UNIVERSITYOF
}

FORWARD

THINKING

WESTMINSTER用

WestminsterResearch

http://www.westminster.ac.uk/westminsterresearch

Influence of fingerprints and finger positioning on accuracy of RF blood glucose measurement from fingertips

Turgul, V. and Kale, I.

This paper is a postprint of a paper submitted to and accepted for publication in Electronics Letters and is subject to Institution of Engineering and Technology Copyright. The copy of record is available at the IET Digital Library:

https://dx.doi.org/10.1049/el.2016.4327

The WestminsterResearch online digital archive at the University of Westminster aims to make the research output of the University available to a wider audience. Copyright and Moral Rights remain with the authors and/or copyright owners.

Whilst further distribution of specific materials from within this archive is forbidden, you may freely distribute the URL of WestminsterResearch: ((http://westminsterresearch.wmin.ac.uk/).

In case of abuse or copyright appearing without permission e-mail repository@westminster.ac.uk 


\section{Influence of fingerprints and finger positioning on accuracy of RF blood glucose measurement from fingertips}

\section{Turgul ${ }^{\bowtie}$ and I. Kale}

Non-invasive blood glucose measurement has attracted great interest from researchers deploying various techniques where microwave sensing is one of them. Microwave resonators are utilised as sensors for measuring the glucose levels. A body part is placed on the sensor for a reading and the measurement principle lies in the change of the dielectric properties of blood with varying levels of glucose. The fingertip is a popular measurement site as there is a good amount of fresh blood supply. The position of the fingertip on the sensor has an effect on the sensor response due to the change in the propagation path of the EM field inside the finger. Moreover, fingerprints also affect the sensor response as the irregular ridges and valleys in the fingerprint introduce air gaps altering the effective permittivity seen by the sensor. The effects of fingerprints as well as finger positioning on the sensor is explored and explained.

Introduction: Diabetes mellitus is a well-known disease associated with high blood glucose levels (BGLs), affecting over 400 million people worldwide and the number keeps increasing [1]. At the moment, the conventional BGL measurement method is the use of portable measurement devices where a small blood sample is needed; therefore, this method is uncomfortable. Hence, there is a great deal of research conducted on non-invasive BGL measurement methods [2]. Microwave sensing is a promising method where microwave resonators are used as the sensing element. A variation in the concentration of the glucose in blood induces a change in the overall dielectric properties of the blood. The sensor's frequency response changes accordingly as a result of this. Preliminary studies show that a frequency shift of $<8 \mathrm{MHz}$ is expected with varying levels of glucose in the blood [3]. The fingertip is the most common site to obtain the blood sample for the conventional method and it is also a desirable measurement site for the non-invasive microwave BGL measurements due to having a lot of fresh blood supply. Furthermore, it is easy to position the finger on the sensor and the fingertip is homogenous in terms of biological layers present. However, a positioning error may introduce more frequency shift than the shift caused by the change in the glucose content. Moreover, the irregular shape of the fingerprint in the fingertip adds to this error. To demonstrate this, simulations were performed with the conventional 4-layer fingertip model as well as a novel model with a fingerprint imprinted. Additionally, the effect of positioning of the finger on the sensor was investigated. There are other irregularities on whichever measurement site used possibly contributing to the measurement error such as hair follicles on skin, sweat ducts and moisture due to sweat which also need to be considered; however, these are not in the scope of this work.

Non-invasive sensor and measurement: A sensitive microwave sensor was designed and used in this Letter with the layout as shown in Fig. 1. The sensor operates at $4.8 \mathrm{GHz}$ in air and $3.25 \mathrm{GHz}$ when a fingertip is placed on top.

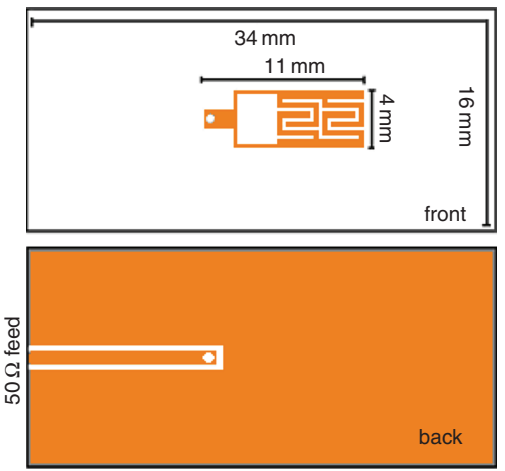

Fig. 1 Layout of sensor used in this Letter

The permittivity of blood decreases as the glucose concentration increases which causes a shift in the resonance frequency. The sensor was tested with glucose/water solutions as an approximation of blood from 100 to $500 \mathrm{mg} / \mathrm{dl}$, which reflects a realistic range. The permittivity difference between the two concentration levels is about 0.24 and the shift in the resonance frequency is about $8 \mathrm{MHz}$. The expected shift is much lower when a fingertip is used due to the lossy skin and fat layers present before the blood layer. For this reason, in order to resolve small variations in the BGL any sources of error must be eliminated or reduced greatly.

Fingertip model: The 4-layer fingertip model was realised in CST Microwave Studio (MWS) with skin, fat, blood and bone layers with the thickness and dielectric constant values as shown in Fig. 2.

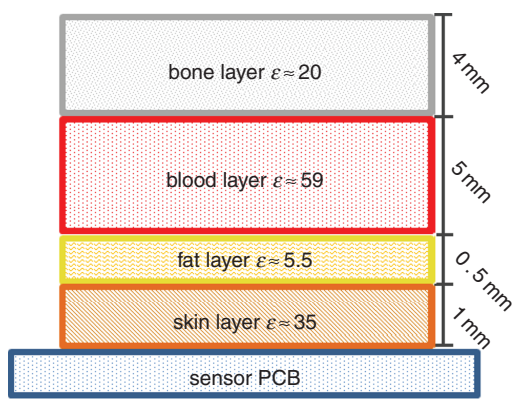

Fig. 2 4-Layer fingertip model layers

Human fingertips are imprinted with fingerprints which are comprised of ridges and valleys with specific patterns to individuals. The valleys are $20 \mu \mathrm{m}$ deep on average. This effectively forms air gaps on the skin when the fingertip is pressed against the sensor, altering the sensor response. The models used in CST MWS are shown in Fig. 3. The fingerprint pattern used was randomly generated that is the representative of a human fingerprint.
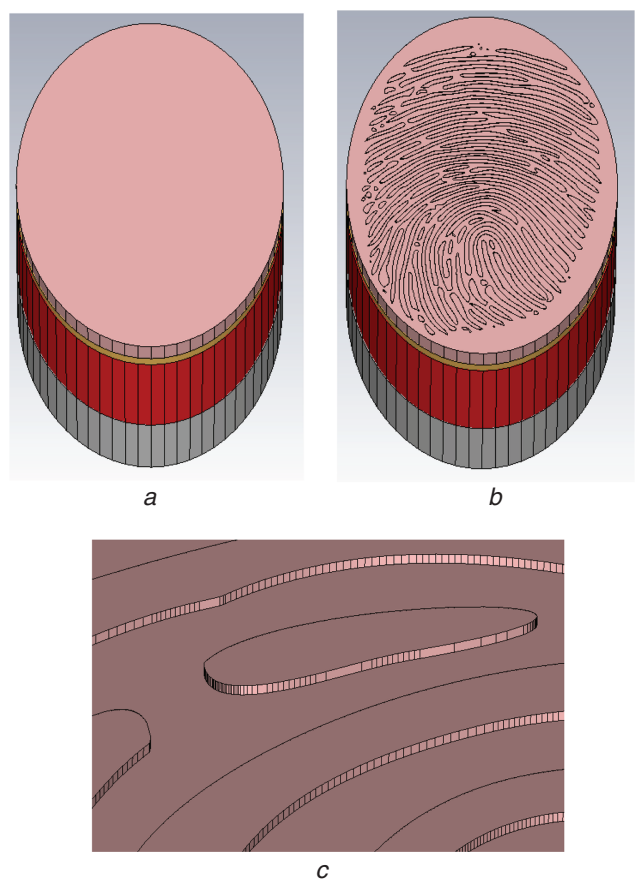

Fig. 3 4-Layer fingertip model realised in CST MWS

a Conventional model

$b$ Model with fingerprin

$c$ Detailed view of section of fingerprint

Results: Two simulations were performed to evaluate the effect of fingerprints and finger positioning on the sensor. First, the sensor was simulated with the conventional fingertip model and then with the model with the fingerprint to compare. The results are shown in Fig. 4. A difference of $14 \mathrm{MHz}$ in the resonance frequency was observed between the two models. With the fingerprint model, the observed resonance frequency is higher due to the valleys forming air gaps and reducing the effective permittivity seen by the sensor. Although the 
air gaps introduced by the fingerprint are quite small structurally, their effect on the sensor's frequency response is considerable as this is the first layer experienced by the EM field. The air gaps prevent uniformly coupling the sensor to the skin. Consequent layers have less effect on the sensor response due to the attenuation in the signal power level caused by the preceding layers.

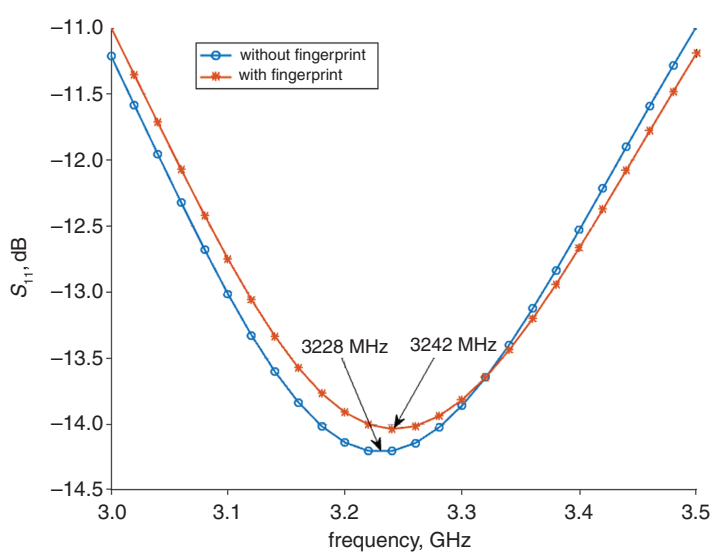

Fig. 4 Resonance frequency of sensor with and without fingerprint

Second, the fingertip's position was varied from -2 to $2 \mathrm{~mm}$ with $0.5 \mathrm{~mm}$ steps in both $x$ and $y$ directions relative to its original position $(0,0)$. This position variation was deemed sufficient since the position can mostly be controlled by using an enclosure to hold the sensor and the fingertip in place eliminating a greater positioning error. The resulting resonance frequency values were normalised to the resonance frequency at the origin and were plotted in MATLAB. The obtained result is shown in Fig. 5.

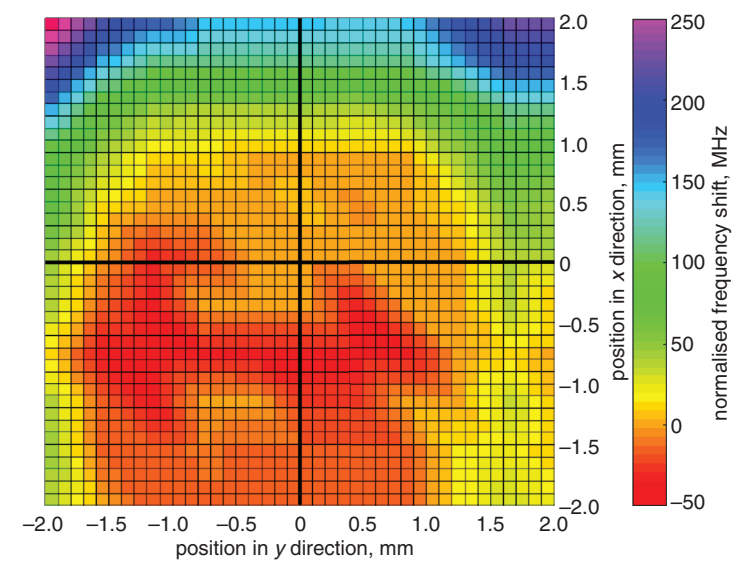

Fig. 5 Normalised frequency shift against fingertip position on sensor

As can be seen from Fig. 5, the position of the fingertip on the sensor affects the resonance frequency where the magnitude of the effect depends on the location. Moreover, the results show that the shift caused is not symmetrical with respect to the origin in any of the quadrants of the graph. This is due to the asymmetrical nature of the fingerprint. A positioning error may cause up to $250 \mathrm{MHz}$ difference in the resonance frequency of the sensor. Considering the small frequency shift expected due to the variation in the BGL, an error of this magnitude may totally result in erroneous readings. Therefore, attention must be paid to the positioning of the fingertip on the sensor where fingerprint increases the level of error due to its irregularity.

Another crucial point to be considered is the pressure applied onto the sensor by the fingertip, which also has a direct effect on the resonance frequency and may cause a difference in the resonance frequency up to $100 \mathrm{MHz}$ as shown by Turgul and Kale in [4]. The reason is that the effective thickness of the layers varies with pressure. Moreover, the pressure variation causes the ridges to squeeze which effectively changes the shape of them and the volume of the air gaps. This ultimately affects the resonance frequency as well. Therefore, increasing the pressure applied to minimise the air gaps is not a solution to achieve a better electrical coupling between the sensor and the skin since it will have an effect on the resonance frequency. Additionally, applying excessive pressure pushes away the blood from the fingertip which in turn reduces the amount of sample to be measured by the sensor. In conclusion, factors such as finger positioning and pressure applied need to be carefully addressed to obtain precise BGL readings where the expected frequency shift is already very small due to blood glucose variation. To this effect, the problem of pressure-dependent fingerprint variations and its effects were investigated and will be reported in another Letter.

Conclusion: The effect and importance of fingerprints and fingertip positioning on non-invasive RF glucose sensors were explained and demonstrated. The frequency shift expected due to the varying BGLs is well below $8 \mathrm{MHz}$, whereas a positioning error may cause an error up to $250 \mathrm{MHz}$ and a pressure variation applied by the fingertip may cause and error up to $100 \mathrm{MHz}$. This could lead to wrong BGL predictions. The irregular shape of the fingerprints makes the readings more prone to positioning errors for the reasons explained previously. We shall in a future publication report on the dynamic compensation for this problem.

(C) The Institution of Engineering and Technology 2017

Submitted: 25 November 2016

doi: $10.1049 / \mathrm{el} .2016 .4327$

One or more of the Figures in this Letter are available in colour online.

V. Turgul and I. Kale (Applied DSP and VLSI Research Group (ADVRG), Department of Engineering, Faculty of Science and Technology, University of Westminster, WIW 6UW London, United Kingdom)

凶E-mail: volkan.turgul@my.westminster.ac.uk

\section{References}

1 International Diabetes Federation (IDF): 'Diabetes atlas' (2015, Seventh edn.), accessed May 2016

2 Vashist, S.: 'Continuous glucose monitoring systems: a review', Diagnostics, 2013, 3, (4), pp. 385-412

3 Turgul, V., and Kale, I.: 'Characterization of the complex permittivity of glucose/water solutions for non-invasive RF/microwave blood glucose sensing'. IEEE Int. Instrumentation and Measurement Technology Conf. (I2MTC), Taipei, May 2016

4 Turgul, V., and Kale, I.: 'A novel pressure sensing circuit for noninvasive RF/microwave blood glucose sensors'. IEEE Mediterranean Microwave Symp. (MMS), Abu Dhabi, November 2016 